\title{
NEUES AUS ITAS
}

\section{New Project: SUSTAINABLE URBAN TOURISM: Involving Local Agents and Partnerships for New Forms of Governance}

\begin{abstract}
The Karlsruhe Research Center, Institute for Technology Assessment and Systems Analysis (ITAS) is pleased to announce the beginning of an international research and policy project on "SUSTAINABLE URBAN TOURISM: Involving Local Agents and Partnerships for New Forms of Governance" (SUT). Our partners in this new initiative are: The Vienna University of Economics and Business Administration and Regional Consulting Ziviltechniker GmbH, Vienna; The Aristotle University of Thessaloniki, Greece; and The Veliko Turnovo University of Bulgaria. We would also like to acknowledge the generous financial support provided by "The Fifth Framework Program" (Key Action "City of Tomorrow and Cultural Heritage") funded by the Commission of the European Communities.
\end{abstract}

\section{Research Objectives}

European cities face high and growing demands from tourism, and the pressures and problems associated with the management of visitors have to be systematically tackled by all parties concerned. SUT presents an international and a multidisciplinary effort to work with publicprivate partnerships and urban governments in Europe to develop, validate, and deploy a general framework for urban sustainable tourism partnerships that is applicable to a variety of urban municipal and development contexts. The overall goal of the project is to elaborate and promote innovative forms and instruments of local governance to improve urban tourism development involving the principles of sustainability and participatory decisionmaking.

\section{Problems to be tackled}

Sustainable urban tourism requires attention to varied elements, including (a) maintaining physical heritage in the context of living, developing cities; (b) allowing maximum access to available infrastructure, tourist sites, parks, and other green spaces; (c) strengthening the cultural and social viability of local community; (d) balancing interests of residents and visitors; (e) economic viability (providing sound longterm development and high quality employment opportunities); (f) minimizing adverse ecological impacts on sites from transportation, and (g) unsustainable consumption patterns. Achieving this involves advancing knowledge and practice about the types of partnerships that can be formed to promote urban sustainable tourism; the roles of government and other stakeholders; how local governments can apply such partnerships to local tourism development; and the critical issues and challenges to local governments seeking to create such partnerships. By providing local governments with a framework to catalyze partnerships, SUT will help them engage principle stakeholders from the private and public sectors to address issues of urban sustainability, challenges, and opportunities. The frameworks and benchmark tools developed by the project will help them realize linkages between tourism and sustainable development, including using the principles of Agenda 21 for tourism decision-making and improved management, planning, and implementation practices.

\section{Geographical focus}

The project's national and case study field research will be focused in Germany, Austria, Greece and Bulgaria. These four countries present a mix of conditions for the analysis of urban sustainable tourism partnerships, with a range of contexts in terms of such factors as levels of economic development, federal v. unified governmental structure, administrative capabilities, roles of local government and other nongovernmental groups, types of tourism, and environmental policy frameworks. The inclusion of Bulgaria - an EU accession candidate is important because this will allow assessment of obstacles and potentials for such countries to 
reach European norms and also to draw on existing European experience. Within the four countries, case study efforts will focus on selected cities. These are Heidelberg (DE), Graz (A), Thessaloniki (Gr), and Turnovo (BG), all of which have been internationally recognized in their sustainable development efforts and have developed tourism as a strategic economic sector.

\section{Expected results and impacts}

This project is designed to advance knowledge, tools, and practices to assist decision-makers, governments, and other stakeholders in urban tourism development, including the private sector and non-governmental groups, to implement sustainable tourism practices. The project's work will contribute to an enhanced understanding of sustainable urban tourism and its practical applications and create knowledge that can help to foster improvements in urban governance in the pursuit of sustainability. The project will also develop improved methodological models and tools, including a unified framework and a widely diffused and customized benchmarking procedure.

Specific research and policy application of the study findings include: (a) Elaboration of the general principles concerning sustainability and tourism development applicable at the local level of urban development; (b) Promotion of alternative practices of urban governance in order to allow flexible adaptation to the different types of problems of urban areas; (c) Enhancement of direct contacts among various levels of governance and especially among same level local actors without passing necessarily through higher level authorities; (d) Facilitation of the communication among partnership members through the homogenization of basic terminology and standardization of main practices; (e) Improvement in the efficiency of resources devoted to the formulation and implementation of sustainable urban tourism; (f) Development of basic sustainability indexes of urban tourism development; (g) Enhancement and distribution of information and experience related to best practices of European urban development.

In practice, establishing private-public partnerships will help the development of sus- tainable tourism facilities in urban areas, develop attractions and activities related to cultural heritage of the built environment and historic districts, promote environmentally friendly modes of transportation and reduce tourismrelated traffic, develop projects to mitigate damage to destroyed environment, provide information to tourists on appropriate behaviour and risks, integrate sustainable development education in local universities and schools and tourism into curriculum, promote networks for information, and research on sustainable tourism.

\section{Project organization}

This three-year project will employ the expertise of scientists and experts from four European countries working in various research fields linking together issues of urban tourism, sustainability, and innovative participatory decision-making. As a lead partner and managing project coordinator, ITAS will be involved in all stages of work, will co-ordinate, and manage all project activities during the entire duration of project. Holding the lead position in this initiative will further endorse the institute's prominent reputation in carrying out successfully large-scale collaborative and interdisciplinary research projects. Moreover, by examining the issues of governance and sustainable urban tourism in relation, SUT will provide new dimensions to ITAS' strong efforts to elaborate innovative research approaches linking together politics, socio-economic development and environmental sustainability. On a different scale, in the arena of assisting public policy, the successful implementation of the project will reinforce ITAS' sound standing among research and technology transfer institutions of various levels, both in Germany and internationally.

To disseminate the methods, results, and recommendations of the project to localities and publicly to the wider set of stakeholders and interested parties, an interactive web site will be developed during the first year of the project.

If you are interested in contacting us or have any inquiries about the project, please email: Krassi@itas.fzk.de or look at ITAS' Webster: http://www.itas.fzk.de/home.htm

(Krassimira Paskaleva

Project Coordinator, ITAS) 


\section{ESTO Launches New Projects to Monitor Technological Future-Oriented Activities}

A project to monitor European technology assessment activities under the leadership of ITAS has recently been launched by the European Science and Technology Observatory (ESTO) network. The project is one of three to monitor EU countries' technology and futureoriented activities. The other two are on technology forecasting, being managed by the VDI Future Technologies division in Düsseldorf, and foresight, being managed by CEST (Centre for Exploitation of Science and Technology, London) and OST (Observatoire des Sciences et des Techniques, Paris).

The project on technology assessment involves a core group of eight institutions from seven countries and aims to track the activities of the most important institutions and programmes for technology assessment in Europe and elsewhere. Based on the results of this tracking activity, topics of special interest will be selected for in-depth analysis by experts. Such topics could be technologies of particular interest, societal issues involving the application of technology, or such aspects as ethics or public participation in decision-making on science and technology related matters. Among the goals of the project are:

- To identify common conclusions from this existing research and to identify areas of uncertainty or controversy regarding the conclusions of this work, in particular with respect to the European dimension of the issue in question.

- To analyse possible reasons for difference in conclusions, e.g. the importance of cultural factors, variety of approaches and methods, country-specific focus on specific concerns.

- While the project will not deal with purely theoretical issues, it will in some cases focus on methods and approaches to examine their suitability for achieving certain goals or in certain decision-making contexts, e.g. the participation of stakeholders and other important actors.
- $\quad$ The work on these analyses may frequently generate ideas for additional research to close gaps in the knowledge, particularly but not exclusively on the European dimension.

- The work on specific topics could lead eventually to the emergence of thematic networks working on the specific topic rather than technology assessment per se.

The results of work will be published on a website, which will probably also provide information on the other two monitoring activities. The coordinates of this web-site will be announced in a subsequent issue of "TA-DatenbankNachrichten".

The European Science and Technology Observatory (http://esto.jrc.es) is a network of institutions with experience in the field of technological assessment at the national level and was formally constituted by the Institute for Prospective Technological Studies (Sevilla, http://www.jrc.es), which belongs to the European Commission's Joint Research Centre.

\section{Contact}

Ingrid von Berg, Michael Rader

Research Centre Karlsruhe

Institute for Technology Assessment and

Systems Analysis (ITAS)

P.O. Box 3640, D-76021 Karlsruhe

Tel.: + 49 (0) 7247-82 3970/2505

Fax: + 49 (0) 7247-82 4806

E-Mail: Berg@itas.fzk.de or rader@itas.fzk.de

$\ll$

\section{Bericht über den ITAS- Workshop: "Environmental Research between Knowledge and Action"}

Am 9. und 10. Dezember 1999 veranstaltete ITAS einen internationalen Workshop zum Thema "Environmental Research between Knowledge and Action" im Forschungszentrum Karlsruhe. Es trugen Experten aus Amerika, England, Holland, Kanada, der Schweiz und Deutschland vor. Im Zentrum des Workshops stand die Frage nach den besonderen Bedin- 
gungen einer sich neu formierenden Umweltforschung, die versucht, die Debatte um "Nachhaltige Entwicklung" und die Ergebnisse der "Global Change-Forschung" zu integrieren. Umweltforschung in diesem Sinne ist problemorientiert und entscheidungsbezogen.

Das eigentlich Innovative findet sich in dem Vernetzungsgedanken, der den Umgang mit Natur zu einem Bestandteil der gesellschaftlichen Gesamtentwicklung macht. Für die Umweltforschung stellt sich damit die Aufgaben, ökologische, soziale und ökonomische Sachverhalte in ihrem Zusammenhang zu erforschen und für politische Entscheidungsträger unter der Perspektive einer dauerhaften und umweltgerechten Entwicklung zu bündeln und entscheidungsbezogen zu präsentieren. Dies zwingt zu einem interdisziplinären und integrativen Ansatz in der Forschung, der sich gleichzeitig dessen bewusst bleiben muss, dass das Wissen für Handlungen $\mathrm{zu}$ organisieren und aufzubereiten ist.

Umweltforschung erhält damit einen zentralen Stellenwert im Zusammenhang mit der Modernisierung der Gesellschaft. Sie hat zum einen die komplexe Beziehung zwischen gesellschaftlicher Entwicklung und Umweltveränderung zu erforschen, zum anderen stellt sich ihr das Problem, dieses Wissen in die Entscheidungsprozesse einzubringen. Ihre Rückbindung an Politik oder andere wichtige Handlungsbereiche macht sie zu einer problemorientierten, praxisbezogenen Wissenschaft.

Unter der Leitung von Gotthard Bechmann (ITAS) und Nico Stehr (Universität Duisburg) wurden auf dem Workshop in vier Sektionen die Entwicklung und Struktur einer sich herausbildenden problemorientierten Forschung diskutiert.

Die erste Sektion war konzeptionellen Fragen der problemorientierten Forschung gewidmet.

Gotthard Bechmann grenzte die problemorientierte Forschung von Grundlagenforschung und angewandter Forschung ab und zeigte ihren eigenständigen Charakter auf, der im Kern zu einem neuen Typ von Forschung führt. Klaus Peter Japp (Universität Bielefeld) thematisierte die Unterscheidung von Wissen/Nichtwissen als einer wichtigen Dimension der Risiko- und Umweltforschung, wobei Nichtwissen eine ambivalente Position zwischen Sicherung der wissenschaftlichen Erkenntnisproduktion und riskantem Entscheiden zukomme.

Nach den Qualitätsstandards einer transdisziplinär operierenden Umweltforschung fragte Armin Grunwald (ITAS) in seinem Vortrag. Neuartige Anforderungen an die Forschung stellten sich hier unter zwei Gesichtspunkten: Relevanz und Kompatibilität mit disziplinärem Wissen. Die besondere Herausforderung liege dabei in der Übersetzung gesellschaftlicher Bedürfnisse, Prioritäten und Erwartungen in forschungsinterne Relevanzentscheidungen und Kompatibilitätsbeurteilungen.

Rainer Grundmann (Aston University, Birmingham) beschrieb die Rolle einer advokatorischen Wissenschaft im Rahmen der Risikodebatte. Er zeigte, dass es wesentlich ist, wer die realitätsdefinierende Rolle in der Risikodebatte spielt: Sind es die Politiker, Vertreter der Wirtschaft, der Umweltverbände oder die Wissenschaftler? Die Wissenschaftler könnten nur dann eine wichtige Rolle spielen, wenn sie sich von partikularen Interessen freihielten und versuchten, im Risikodiskurs das "öffentliche Interesse" zu vertreten.

Die zweite Sektion befasste sich mit methodischen Fragen einer problemorientierten Umweltforschung.

John Robinson und James Tansey (University of British Columbia, Canada) berichteten über ein Projekt, das sich mit der Zukunft der Georgia Basin Region für die nächsten vierzig Jahre beschäftigte. Ziel des Projektes ist es, in einem Szenario öffentliche Werte, Präferenzen, Meinungen der Bevölkerung mit wissenschaftlichem und anderem Expertenwissen zu integrieren und zu simulieren, um dadurch die Öffentlichkeit in politische Entscheidungsprozesse über die zukünftige Entwicklung einzubeziehen.

Am Beispiel der Klimafolgenforschung diskutierte Fritz Reusswig (PIK Potsdam), dass die Unterscheidung "mehr wissenschaftszentrierte" oder "stärker handlungsbezogene" Forschung an der Realität vorbei geht. Die Klimaforschung habe gezeigt, dass gesellschaftliche Probleme, die als wissenschaftlich bearbeitbar und sozial als lösungsbedürftig definiert werden, ein komplexes Beziehungsgeflecht zwischen Wissenschaft, Politik und Öffentlichkeit voraussetzen, bei dem wissenschaftliche Problemdefinition, gesellschaftliche Werte und 
Konsensbildung miteinander verzahnt sind. Das Intergovernmental Panel on Climate Change (IPPC) sei hierfür ein besonders wichtiges Beispiel.

Hadi Dowlatabati (Carnegie Mellon University, Pittsburg) entwickelte einen integrierten Ansatz für Umweltforschung und -management. Das Verständnis der Umwelt ist - so seine These - eng an unser Handeln gebunden, so dass wissenschaftliche Forschung immer die Folgen des Handelns als konstitutives Merkmal mit in die Untersuchung einbeziehen müsse. Sie werde dadurch zur reflexiven Forschung, die transdisziplinär und problemorientiert vorgehe und ihre Stoppregeln in einem pragmatischen Verständnis des Handelns finden müsse.

Rene v. Schomberg (Tilburg University) beschäftigte sich mit dem Zustandekommen von multilateralen Umweltvereinbarungen. Er zeigte, wie im Rahmen der UN bei gelungener Kompromissbildung mit Hilfe der Wissenschaft teilweise ein gemeinsames Vorgehen zwischen den Vertretern der Umweltverbände (NGOs), der World Trade Organisation (WTO) und der nationalen Regierungen möglich ist.

Die dritte Sektion beschäftigte sich mit der Funktion des Leitbilds "Sustainable Development" im Rahmen der Umweltforschung.

Karl-WernerBrand (TU-München) betonte in seinem Vortrag, dass Nachhaltigkeitsforschung neben ihrer Problemorientierung auch einen starken Handlungsbezug aufweise, der sie zwinge, Wissen unter Unsicherheit zu produzieren. Darüber hinaus sei sie - bedingt durch die Normativität des Leitbildes "Sustainable Development" - zusätzlich in den gesellschaftlich offenen Definitions- und Bewertungsprozess eingebunden. So treten zu kognitiven auch noch soziale und normative Unsicherheiten, die nur in einer transdisziplinären, dialogischen Forschungspraxis bewältigt werden könnten.

An zwei Beispielen (Solarstrombörse und $\mathrm{CO}_{2}$-Gesetzgebung in der Schweiz) diskutierten Priska Gisler und Christian Pohl (ETH Zürich) die Umsetzungsbedingungen des Leitbildes des Sustainable Development. Es gäbe nur drei relevante Aspekte bei der Realisierung: Das Sustainable Development-Konzept müsse in den Kontext des Projektes übersetzt werden und gleichzeitig die differenten Ziele bündeln; es müsse mit den öffentlich relevanten Themen verbunden werden; und es müsse schließlich den dynamischen Entwicklungen des Anwendungsbereichs angepasst werden.

Die Frage, warum in den siebziger Jahren während der beiden Ölkrisen trotz wirtschaftlicher Stagnation der Energiekonsum und die Umweltzerstörung gewachsen sind, versuchte Ottmar Edenhofer (TU Darmstadt) anhand eines Computermodells zu erklären. Dieses Modell setzt den ökonomischen, sozialen und ökologischen Wandel in Beziehung zueinander und versucht, die wechselseitigen Abhängigkeiten in der Zeit darzustellen und zu simulieren.

Die vierte Sektion thematisierte die Rolle der Wissenschaft in den Medien und in der Öffentlichkeit.

Hans v. Storch (GKSS Geesthacht) stellte Ergebnisse einer empirischen Studie vor. 1000 Wissenschaftler in den USA, Kanada und Deutschland wurden mit Hilfe eines Fragebogens über ihre Meinungen und Einstellungen zum Klimawandel befragt. Als einer der wesentlichen Befunde ließe sich feststellen, dass länderübergreifend viele Forscher glauben, dass die Klimawissenschaft die Objektivität einer wertneutralen Wissenschaft verloren hat. Dies könne mit dem soziokulturellen Kontext zusammenhängen, in dem jede Forschergemeinde sich befindet, sowie mit dem politischen Charakter der Klimawissenschaft und ihrem relativ kurzen Bestehen. Wenn die Wissenschaft ihren wertneutralen und objektiven Charakter verlöre, werde ein Großteil des Wissens, welches an die Öffentlichkeit gelangt, gleichermaßen zum Gegenstand von sozialen Interpretationen wie von gesicherten Fakten. Klima als Gegenstand der Forschung stelle sich als ein soziales Konstrukt heraus, an dessen Definition Wissenschaft, Öffentlichkeit und Massenmedien beteiligt seien.

Klaus Eder (Humboldt Universität, Berlin) entwickelte einen analytischen Rahmen, um die Beziehung zwischen Öffentlichkeit und Wissenschaft zu beschreiben. Er zeigte durch eine Analyse der ökologischen Debatte, dass trotz selektiven und manchmal auch falschen Gebrauchs der Wissenschaft durch die Öffentlichkeit diesem Wechselspiel doch eine kollektive Rationalität zu Grunde liegt. Je mehr die Wissenschaft und die Öffentlichkeit die Logik ihres eigenen Diskurses verfolgten, um so stärker seien sie aufeinander angewiesen. 
Mit der ambivalenten Rolle der Wissenschaft in der Öffentlichkeit befasste sich Jost Halfmann (TU Dresden). Obwohl die Wissenschaft ihre Aura und Autorität im Laufe der ökologischen Debatte als Produzent von "wahrem" Wissen verloren habe, werde doch immer auf Wissenschaft zugegriffen, wenn sich die gesellschaftlichen Institutionen mit neuen Risiken oder Umweltproblemen konfrontiert sehen. Gerade die zunehmende Nachfrage nach Wissen seitens der gesellschaftlichen Entscheidungsträger bringe die Wissenschaft in das Dilemma, mehr an Lösungskapazität versprechen zu müssen, als auf Grund der Komplexität der zu entscheidenden Probleme wissenschaftlich vertretbar und machbar sei. Sowohl die Zeit als auch die finanziellen Ressourcen der Wissenschaft seien auf dem Feld der ökologischen Forschung begrenzt.

\section{Fazit}

Der Workshop hat deutlich gemacht, dass sich außerhalb des traditionellen Wissenschaftssystems eine nicht disziplinäre Forschung zu formieren beginnt, die abweichend von den bisherigen Standards der Wissenschaft Wissen produziert, das durch wachsende öffentliche Nachfrage stimuliert wird. Indem solches Wissen in Wirtschaft, Politik und Kultur nachgefragt wird und in diesen Systemen Leistungen erbringen soll, gewinnt der Kontext wissenschaftlichen Wissens zumindest in dreifacher Hinsicht an Bedeutung:

- Wissenschaft ändert ihren Legitimationsmodus. Gemeint ist damit, dass nicht mehr allein die "Objektivität des Wissens" für ihre Legitimation ausschlaggebend ist, sondern die Bedeutung und der praktische Nutzen für ihre Anwender in den unterschiedlichen gesellschaftlichen Bereichen wird ein Kriterium ihrer Qualität.

- Wissenschaft wird selbstreflexiv. Das bedeutet, sie wird gezwungen, über ihre Anwendungsbedingungen und Anwendungserfolge oder Misserfolge nachzudenken. Wissenschaft selbst wir zum Gegenstand wissenschaftlicher Forschung (Risikoforschung, Technikfolgenforschung usw.)
- Wissenschaft erzeugt und macht Nichtwissen bewusst. Gemeint ist damit, dass gerade auf dem Gebiet der Umweltforschung gesicherte Aussagen über problematische, gesellschaftlich zu steuernde Kausalbeziehungen auf Grund komplexer Verursacherkonstellationen nicht leicht zu machen sind. Forschung erzeugt neuen Bedarf an Forschung.

Mit der Umweltforschung, der Risikoforschung oder der Global Change-Forschung sind nicht nur neue Formen der Wissensproduktion entstanden, sondern es ist eine Veränderung der gesellschaftlichen Funktion der Produktion wissenschaftlichen Wissens zu registrieren, die sowohl transdisziplinäre Arbeitsweisen wie auch neue theoretische Konzepte erfordert. Man könnte hier auch von einem neuen Typ von Forschung sprechen, der neben der Grundlagenforschung und der angewandten Forschung als "problemorientierte Forschung" zunehmend gesellschaftliche Relevanz gewinnt.

Die Beiträge des Workshops werden für eine Publikation im Herbst vorbereitet, auf deren Erscheinen in den TA-DatenbankNachrichten hingewiesen werden wird.

(Gotthard Bechmann)

\section{Teilnehmerinnen und Teil- nehmer bei der Tagung "Trans- sektorale Forschung zum Glo- balen Wandel"}

Ca. 150 Teilnehmerinnen und Teilnehmer konnte Prof. Dr. Manfred Popp, Forschungszentrum Karlsruhe, am 27. Januar 2000 in Bonn bei der vom ITAS gemeinsam mit der Europäischen Akademie zur Erforschung von Folgen wissenschaftlich-technischer Entwicklungen, Bad Neuenahr-Ahrweiler, und der Akademie für Technikfolgenabschätzung in BadenWürttemberg, Stuttgart, organisierten Tagung "Transsektorale Forschung zum Globalen Wandel" begrüßen. Im Plenum und in parallelen Arbeitsgruppensitzungen wurden drei Beispiele transsektoraler Forschungsansätze bzw. -projekte: 
- das Syndrom-Konzept des Wissenschaftlichen Beirats der Bundesregierung Globale Umweltveränderungen (WGBU)

- BMBF-Leitprojekt "AQUA: Availability, Quality and Allocation" im Rahmen des Förderprogramms "Globaler Wandel des Wasserkreislaufes (GLOWA)" und

- das Strategiefondsprojekt der HelmholtzGemeinschaft: Global zukunftsfähige Entwicklung - Perspektiven für Deutschland

vorgestellt, durch neun eingeladene Diskussionsbeiträge ergänzt und intensiv diskutiert.

Wir werden auf die Ergebnisse der Tagung anlässlich der Veröffentlichung des Tagungsbandes zurückkommen, der im Herbst beim Campus-Verlag in der Schriftenreihe des Instituts für Technikfolgenabschätzung und Systemanalyse erscheinen wird.

(Reinhard Coenen)

$\gg$

\section{Neue Veröffentlichung: "Stoff- ströme und Kosten in den Be- reichen Bauen und Wohnen"}

Die von ITAS zusammen mit dem Institut für Industrielle Bauproduktion (ifib) für die Enquete-Kommission Schutz des Menschen und der Umwelt des 13. Deutschen Bundestages durchgeführte Studie "Stoffströme und Kosten in den Bereichen Bauen und Wohnen" liegt jetzt auch als Buchveröffentlichung beim Springer-Verlag vor.

Im Bereich Bauen und Wohnen fallen große Teile der gesellschaftlich erzeugten Stoffströme und damit verbundene Umweltbelastungen an. Das Verhältnis zwischen Stoffeintrag und Abfall liegt je nach Systemgrenzen zwischen 3:1 und 5:1. Durch die vergleichsweise hohe durchschnittliche Lebenserwartung von Bauten und Bauteilen werden die heute verbauten Stoffe erst mit einer Verzögerung von 50 bis 100 Jahren als Abrissmaterialien und potentieller Müll anfallen. Der Baubereich stellt daher ein riesiges "Zwischenlager" dar. Dieses Lager können wir entweder betrachten als Zwischendeponie für die Abfallmengen der Zukunft oder als Ressource für die Baustoffe der $\mathrm{Zu}$ kunft.
Die Studie beruht auf der Kombination von makroökonomischen Stoffflussanalysen und einem dynamischen Lebenszyklusmodell des Gebäudebestandes. Die vorgelagerten Prozesse und die möglichen Auswirkungen von Problemstoffen in Nutzung und Recycling wurden zusätzlich berücksichtigt.

(Reinhard Coenen)

\section{Bibliographische Angaben:}

Kohler, Niklaus; Hassler, Uta; Paschen, Herbert (Hrsg.): Stoffströme und Kosten in den Bereichen Bauen und Wohnen, Springer-Verlag 1999. 301 S. DM 98,--. ISBN 3-540-66073-9

\section{Polnischer Wissenschaftler ist Gast des ITAS}

Herr Professor Dr. phil. habil. Andrzej Kiepas, Direktor des Instituts für Philosophie der Schlesischen Universität Katowice (Polen), wird vom 08. März bis zum 07. April 2000 Gastwissenschaftler des ITAS sein. Der Gastaufenthalt wird einerseits dazu dienen, Material für ein in Polen herauszugebendes Buch zum Thema "Rationalität in Wissenschaft und Technik" zu sammeln sowie bestehende Forschungskontakte zu vertiefen und neue Kooperationen anzuknüpfen. ITAS erwartet andererseits von Herrn Kiepas einen Know-how-Transfer auf folgenden Gebieten: Stand der philosophischen Diskussion zu Fragen der Rationalität in Wissenschaft und Technik in Polen, Überlegungen zur Institutionalisierung von Technikfolgenabschätzung in der Lehre auf dem Gebiet der Philosophie und der Ingenieurwissenschaften in Polen und in Deutschland, Anregungen zur weiteren Kooperation zwischen ITAS und polnischen TAEinrichtungen sowie Informationen über die inhaltliche und organisatorische Vorbereitung eines geplanten Workshops im Mai 2000 in Wisla (Polen). Mit diesem Know-how wird ITAS in die Lage versetzt, seinen Kooperationsbemühungen und -verpflichtungen gegenüber seinen Partnern in Ländern Ostmittel- und Mitteleuropas gezielter nachzukommen, um so besser bi- bzw. multilaterale Projekte vorbereiten und realisieren zu können.

(Gerhard Banse) 


\section{Hinweis der Redaktion}

Zwei weitere Beiträge mit Informationen zu den Aktivitäten des ITAS finden sich in anderen Rubriken der TA-Datenbank-Nachrichten:

- Zum einen ist dies eine Darstellung des ITAS-Projektes "Energetische Nutzung biogener Abfälle" im Schwerpunkt dieses Heftes (S. 53 ff), das Anfang des Jahres 1999 begonnen wurde.

- $\quad$ Eine Rezension des von Norman Vig und Herbert Paschen herausgegebenen Sammelbandes "Parliaments and Technology. The Development of Technology Assessment in Europe" ist auf Seite 96 bei den TA-relevanten Büchern zu finden.

$\ll \gg$ 